INTER NATIONAL MONETARY FUND

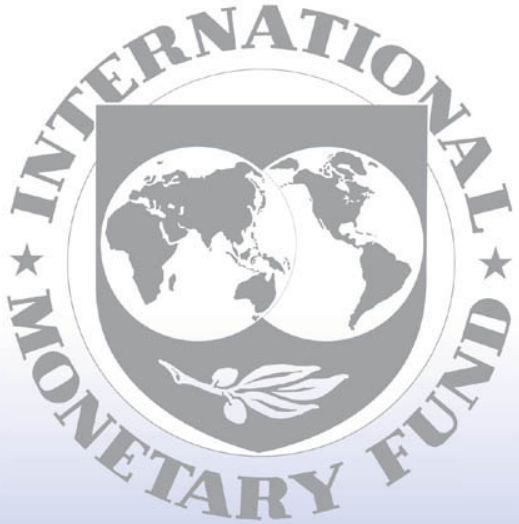

Staff

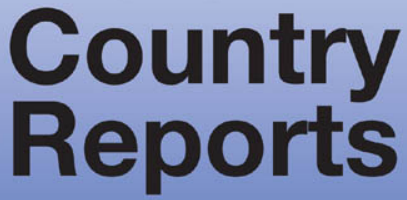




\section{St. Kitts and Nevis: Statistical Appendix}

This Statistical Appendix for St. Kitts and Nevis was prepared by a staff team of the International Monetary Fund as background documentation for the periodic consultation with the member country. It is based on the information available at the time it was completed on December 17, 2007. The views expressed in this document are those of the staff team and do not necessarily reflect the views of the government of St. Kitts and Nevis or the Executive Board of the IMF.

The policy of publication of staff reports and other documents by the IMF allows for the deletion of market-sensitive information.

Copies of this report are available to the public from

International Monetary Fund $\bullet$ Publication Services

700 19th Street, N.W. • Washington, D.C. 20431

Telephone: (202) 623-7430 • Telefax: (202) 623-7201

E-mail: publications@imf.org • Internet: http://www.imf.org

Price: $\$ 18.00$ a copy

\section{International Monetary Fund Washington, D.C.}


This page intentionally left blank 


\section{INTERNATIONAL MONETARY FUND}

\section{ST. KITTS AND NEVIS}

\section{Statistical Appendix}

Prepared by Nancy Wagner, Hunter Monroe, Nkunde Mwase, and Yan Sun

Approved by the Western Hemisphere Department

December 17, 2007

Contents

Basic Data

Tables

1. GDP by Sector at Constant Prices

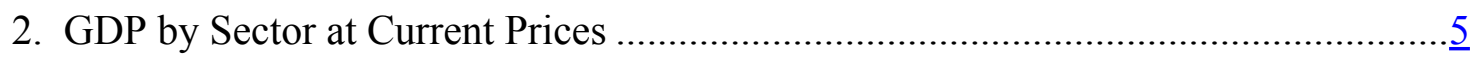

3. Selected Data on Tourism ………….............................................................

4. Selected Indicators of Agricultural Production ………………................................

5. Average Annual Wage Rate of Workers .............................................................

6. Number of Workers in the Social Security Scheme by Age Group ………...............

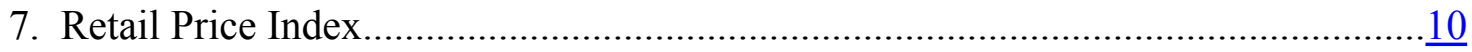

8. Central Government Operations .....................................................................

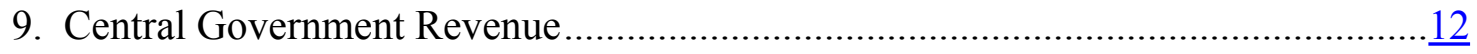

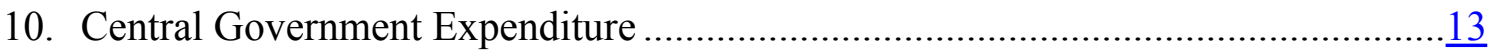

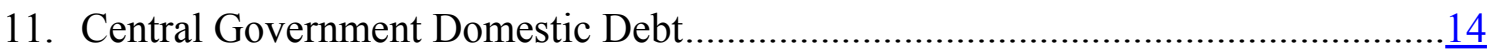

12. Operations of the Social Security Board............................................................

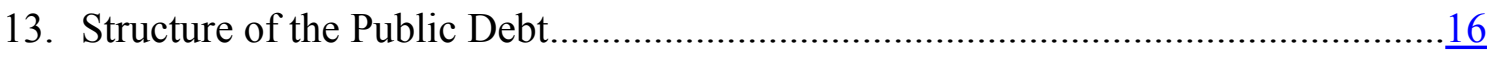

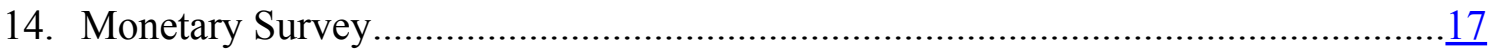

15. Eastern Caribbean Central Bank Local Operations ................................................ 18

16. Consolidated Balance Sheet Accounts of the Commercial Banks ............................19

17. Sectoral Distribution of Commercial Bank Loans .................................................

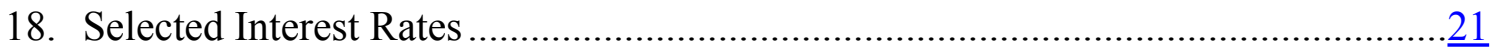

19. Selected Performance Indicators of the Commercial Banks....................................22

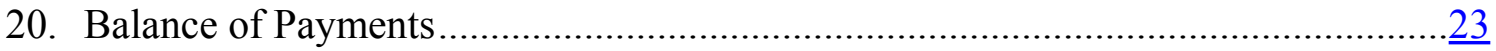

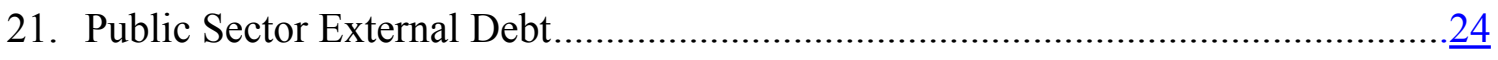


St. Kitts and Nevis: Basic Data

I. Social and Demographic Indicators

Area (sq. km)

Population

Total (thousands, 2001)

Rate of growth (percent per year, 2001)

Density (per sq. km., 2001)

Net migration rate (per thousand, 2002)

Population characteristics

Life expectancy at birth (years, 2005)

Infant mortality (per thousand live births, 2005)

Under 5 mortality rate (per thousand, 2004)

$\begin{aligned} 269.4 & \text { Adult literacy rate (percent, 2004) } \\ & \text { Health and nutrition } \\ 46.1 & \text { Calorie intake (per capita a day, 1996) } \\ 0.02 & \text { Population per physician (thousand, 1993) } \\ 171.2 & \text { Access to safe water (percent, 1994) } \\ -9.8 & \text { AIDS incidence rate (per 100,000, 1998) } \\ & \text { Gross Domestic Product (2006) } \\ 71 & \text { (millions of U.S. dollars) } \\ 18 & \text { (millions of E.C. dollars) } \\ 18 & \text { (US\$ per capita) }\end{aligned}$

II. Economic Indicators, 2002-06

2003

2004

2005

Est.

(In percent of GDP)

\section{Origin of GDP at factor cost}

Agriculture and mining

Manufacturing and construction

Services and others

$\begin{array}{rrrrr}3.8 & 3.4 & 3.5 & 3.4 & 2.9 \\ 26.4 & 25.2 & 24.6 & 23.3 & 23.2 \\ 69.8 & 71.5 & 71.9 & 73.3 & 73.9\end{array}$

71.9

73.3

(Annual percentage changes, unless otherwise specified)

National accounts and prices

Real GDP

GDP deflator

Consumer price index (period average)

Consumer price index (end of period)

$-0.3 \quad-1.2$

1.5

2.1

1.8

2.7

2.3

2.9

(In percent of GDP)

Gross domestic investment

Of which:

Central government

Gross national savings

External savings

Consumption

Of which

Central government consumption

$\begin{array}{rrrrr}51.8 & 45.6 & 43.8 & 42.4 & 54.0 \\ 14.0 & 7.0 & 6.2 & 6.7 & 7.6 \\ 12.7 & 10.7 & 23.6 & 19.8 & 25.0 \\ 39.1 & 34.9 & 20.2 & 22.6 & 29.0 \\ 78.3 & 79.5 & 68.2 & 73.7 & 69.1 \\ & & & & \\ 23.5 & 22.8 & 23.7 & 23.5 & 22.3\end{array}$

Public finances

Central government

Total revenue and grants

Total expenditure and net lending

Of which

Interest

Current balance

Primary balance

Overall balance

Total public sector debt, in percent of GDP 1/

$\begin{array}{rrrrr}34.5 & 33.5 & 34.3 & 39.5 & 38.9 \\ 53.9 & 42.5 & 42.1 & 43.5 & 44.2 \\ 7.4 & & & & \\ -2.9 & 7.6 & 7.1 & 8.1 & 9.6 \\ -12.0 & -1.2 & -1.0 & -0.1 & 0.3 \\ -19.4 & -1.4 & -0.8 & 4.0 & 4.3 \\ 163.0 & -9.0 & -7.9 & -4.1 & -5.3 \\ & 181.5 & 195.0 & 194.8 & 184.6\end{array}$

(Twelve-month percentage changes, unless otherwise specified)

Money and credit

Broad money

Of which

Money

Quasi money

Net domestic assets of the banking system 2/

Of which

Credit to the public sector (net) $2 /$

Credit to the private sector $2 /$

Broad money, in percent of GDP

Lending interest rate (average, in percent)

$\begin{array}{rrrrr}6.4 & 6.9 & 22.1 & 4.3 & 12.8 \\ 12.9 & & & & \\ 5.4 & 11.0 & 25.4 & -1.9 & 12.7 \\ -3.9 & 6.2 & 21.6 & 5.5 & 12.8 \\ & -6.7 & 27.8 & -4.1 & 5.7 \\ 1.6 & & & & \\ -2.1 & -10.8 & 18.3 & 9.2 & 6.4 \\ 89.2 & 3.4 & 6.8 & 5.1 & 8.2 \\ 10.4 & 92.4 & 102.3 & 97.3 & 97.1 \\ & 12.0 & 9.9 & 9.7 & 9.4\end{array}$


St. Kitts and Nevis: Basic Data

\begin{tabular}{|c|c|c|c|c|c|}
\hline & 2002 & 2003 & 2004 & 2005 & $\begin{array}{l}\text { Est. } \\
2006\end{array}$ \\
\hline \multicolumn{6}{|c|}{ (In millions of U.S. dollars, unless otherwise specified) } \\
\hline \multicolumn{6}{|l|}{ Balance of payments } \\
\hline Current account & -137.2 & -126.3 & -80.5 & -99.1 & -143.8 \\
\hline Merchandise trade balance & -117.2 & -118.6 & -102.2 & -130.7 & -159.6 \\
\hline Exports & 60.4 & 57.0 & 58.6 & 54.5 & 58.6 \\
\hline Imports & -177.6 & -175.6 & -160.8 & -185.2 & -218.2 \\
\hline Services and transfers (net) & -20.0 & -7.7 & 21.7 & 31.6 & 15.8 \\
\hline \multicolumn{6}{|l|}{ Of which } \\
\hline Public sector interest payment & -16.8 & -20.7 & -21.2 & -22.5 & -22.2 \\
\hline Tourism receipts & 57.1 & 75.4 & 102.6 & 114.5 & 116.3 \\
\hline Capital and financial account & 132.4 & 112.1 & 88.2 & 55.4 & 142.4 \\
\hline Official capital (net) & 53.0 & 54.7 & 22.9 & 14.5 & -0.1 \\
\hline Foreign direct investment & 79.8 & 75.6 & 46.1 & 85.5 & 199.7 \\
\hline Other capital (net) & -0.4 & -18.2 & 19.2 & -44.6 & -57.2 \\
\hline Errors and omissions or external financing gap & 15.7 & 13.6 & 6.3 & 36.9 & 18.4 \\
\hline Change in net foreign assets & 10.9 & -0.7 & 14.0 & -6.8 & 17.1 \\
\hline Merchandise exports (in percent of GDP) & 17.2 & 15.7 & 14.7 & 12.4 & 11.8 \\
\hline Merchandise imports (in percent of GDP) & -50.6 & -48.5 & -40.3 & -42.3 & -44.1 \\
\hline Current account (in percent of GDP) & -39.1 & -34.9 & -20.2 & -22.6 & -29.0 \\
\hline $\begin{array}{l}\text { Merchandise exports (in U.S. dollars, annual } \\
\text { percentage change) }\end{array}$ & 9.7 & -5.6 & 2.8 & -7.0 & 7.6 \\
\hline $\begin{array}{l}\text { Merchandise imports (in U.S. dollars, annual } \\
\text { percentage change) }\end{array}$ & 6.6 & -1.1 & -8.4 & 15.2 & 17.8 \\
\hline Terms of trade (annual percentage change) & 0.2 & 0.2 & -3.6 & -5.9 & -5.7 \\
\hline $\begin{array}{l}\text { Real effective exchange rate (12-month percentage } \\
\text { change) } 3 /\end{array}$ & -3.3 & -3.9 & -4.0 & 5.4 & 0.5 \\
\hline \multicolumn{6}{|l|}{$\begin{array}{l}\text { International reserve position and } \\
\text { external debt (as of December 31) }\end{array}$} \\
\hline $\begin{array}{l}\text { Total ECCB net international reserves } \\
\text { Of which }\end{array}$ & 501.9 & 536.7 & 630.7 & 596.9 & 693.3 \\
\hline St. Kitts and Nevis imputed net international reserves & 65.4 & 71.5 & 60.5 & 71.5 & 88.6 \\
\hline St. Kitts and Nevis commercial banks' net foreign assets & 51.1 & 94.3 & 61.3 & 102.6 & 115.7 \\
\hline Outstanding external public debt, in percent of GDP & 75.9 & 88.5 & 85.5 & 77.0 & 66.9 \\
\hline External public debt service ratio $4 /$ & 21.0 & 25.5 & 26.2 & 24.1 & 25.1 \\
\hline \multicolumn{6}{|l|}{ Of which } \\
\hline Interest 4/ & 10.8 & 12.1 & 10.5 & 10.3 & 9.7 \\
\hline \multicolumn{6}{|l|}{ IMF data (as of October 31,2007 ) } \\
\hline Membership status: & & & & & Article VIII \\
\hline Intervention currency and rate & & & & & $E C \$ 2.70=U S \$ 1$ \\
\hline Quota & & & & & SDR 8.90 million \\
\hline Fund holdings of national currency & & & & & SDR 8.82 million \\
\hline (as percent of quota) & & & & & 99.09 percent \\
\hline Outstanding purchases and loans: & & & & & none \\
\hline SDR department: holdings & & & & & SDR 0.00 million \\
\hline
\end{tabular}

Sources: St. Kitts and Nevis authorities, Eastern Caribbean Central Bank, PAHO, World Bank, World Health Organization and Fund staff estimates.

1/ All external and domestic debt of the central government, Nevis Island Administration, and public enterprises.

$2 /$ In percent of broad money at the beginning of the period.

3/ End of period. Weights given by the average trade share during 1999-2003.

4/ In percent of exports of goods and services. 
Table 1. St. Kitts and Nevis: GDP by Sector at Constant Prices

\begin{tabular}{|c|c|c|c|c|c|}
\hline & 2002 & 2003 & 2004 & 2005 & $\begin{array}{r}\text { Est. } \\
2006\end{array}$ \\
\hline \multicolumn{6}{|c|}{ (In millions of Eastern Caribbean dollars at 1990 prices) } \\
\hline GDP at market prices & 664.6 & 667.8 & 718.8 & 753.1 & 801.1 \\
\hline Indirect taxes & 102.0 & 112.3 & 122.6 & 130.9 & 154.0 \\
\hline GDP at factor cost & 562.5 & 555.5 & 596.2 & 622.2 & 647.1 \\
\hline Primary sector & 34.8 & 29.4 & 32.8 & 32.1 & 25.9 \\
\hline Agriculture & 31.4 & 27.5 & 30.8 & 30.0 & 23.6 \\
\hline Sugarcane & 12.6 & 9.4 & 9.4 & 7.9 & 0.0 \\
\hline Other crops & 6.4 & 6.5 & 6.9 & 6.2 & 7.3 \\
\hline Livestock & 3.3 & 3.4 & 3.8 & 3.8 & 3.8 \\
\hline Forestry & 0.5 & 0.5 & 0.5 & 0.5 & 0.5 \\
\hline Fishing & 8.7 & 7.8 & 10.2 & 11.6 & 12.0 \\
\hline Mining and quarrying & 3.4 & 1.8 & 2.0 & 2.1 & 2.2 \\
\hline Secondary sector & 174.8 & 161.9 & 165.8 & 169.0 & 173.9 \\
\hline Manufacturing & 73.4 & 73.2 & 76.0 & 78.3 & 75.0 \\
\hline Sugar & 8.1 & 6.2 & 5.4 & 4.1 & 0.0 \\
\hline Other & 65.3 & 67.1 & 70.6 & 74.2 & 75.0 \\
\hline Construction & 101.4 & 88.6 & 89.7 & 90.7 & 98.9 \\
\hline Services & 352.9 & 364.3 & 397.7 & 421.1 & 447.3 \\
\hline Wholesale and retail trade & 67.1 & 70.3 & 69.4 & 70.9 & 77.0 \\
\hline Hotels and restaurants & 23.8 & 31.2 & 40.6 & 44.0 & 45.8 \\
\hline Transport & 45.9 & 45.7 & 61.2 & 62.1 & 62.8 \\
\hline Communications & 55.6 & 53.2 & 55.0 & 64.9 & 72.6 \\
\hline Electricity and water & 11.6 & 12.8 & 13.9 & 14.0 & 15.1 \\
\hline Banking and insurance & 71.0 & 72.6 & 85.2 & 93.0 & 99.2 \\
\hline Real estate and housing & 15.5 & 15.8 & 16.1 & 16.2 & 17.2 \\
\hline Government services & 89.3 & 88.5 & 90.8 & 93.9 & 97.2 \\
\hline Other services & 21.9 & 23.7 & 24.5 & 25.8 & 27.4 \\
\hline \multirow[t]{2}{*}{ Less: Imputed service charge } & -48.9 & -49.5 & -58.9 & -63.7 & -66.9 \\
\hline & \multicolumn{3}{|c|}{ (Percentage changes) } & & \\
\hline GDP at factor cost & -0.3 & -1.2 & 7.3 & 4.4 & 4.0 \\
\hline Primary sector & 13.9 & -15.7 & 11.6 & -2.1 & -19.3 \\
\hline Agriculture & 13.3 & -12.4 & 11.7 & -2.5 & -21.2 \\
\hline Sugarcane & 7.5 & -25.5 & -0.1 & -15.7 & -100.0 \\
\hline Other crops & 33.6 & 2.0 & 6.3 & -10.1 & 18.2 \\
\hline Livestock & 19.6 & 3.3 & 10.9 & 0.8 & -0.5 \\
\hline Forestry & 2.2 & 2.2 & 2.0 & 2.0 & 2.0 \\
\hline Fishing & 8.2 & -10.8 & 31.5 & 13.3 & 3.4 \\
\hline Mining and quarrying & 19.4 & -46.3 & 10.1 & 9.0 & 10.0 \\
\hline Secondary sector & -5.0 & -7.4 & 2.4 & 2.0 & 2.9 \\
\hline Manufacturing & -4.0 & -0.2 & 3.8 & 3.0 & -4.2 \\
\hline Sugar & -4.9 & -24.0 & -12.8 & -24.2 & -100.0 \\
\hline Other & -3.9 & 2.7 & 5.3 & 5.1 & 1.0 \\
\hline Construction & -5.6 & -12.6 & 1.3 & 1.1 & 9.0 \\
\hline Services & 0.9 & 3.2 & 9.2 & 5.9 & 6.2 \\
\hline Wholesale and retail trade & 0.3 & 4.7 & -1.3 & 2.1 & 8.6 \\
\hline Hotels and restaurants & -3.6 & 31.3 & 29.9 & 8.6 & 4.0 \\
\hline Transport & 7.9 & -0.5 & 34.1 & 1.5 & 1.0 \\
\hline Communications & -6.8 & -4.4 & 3.4 & 18.1 & 11.8 \\
\hline Electricity and water & 9.8 & 10.6 & 8.3 & 0.8 & 7.6 \\
\hline Banking and insurance & 1.8 & 2.2 & 17.3 & 9.2 & 6.7 \\
\hline Real estate and housing & 1.0 & 2.0 & 1.5 & 1.0 & 6.0 \\
\hline Government services & 3.8 & -1.0 & 2.6 & 3.4 & 3.5 \\
\hline Other services & 1.4 & 8.1 & 3.4 & 5.1 & 6.5 \\
\hline
\end{tabular}

Sources: St. Kitts and Nevis authorities; and Fund staff estimates. 
Table 2. St. Kitts and Nevis: GDP By Sector at Current Prices

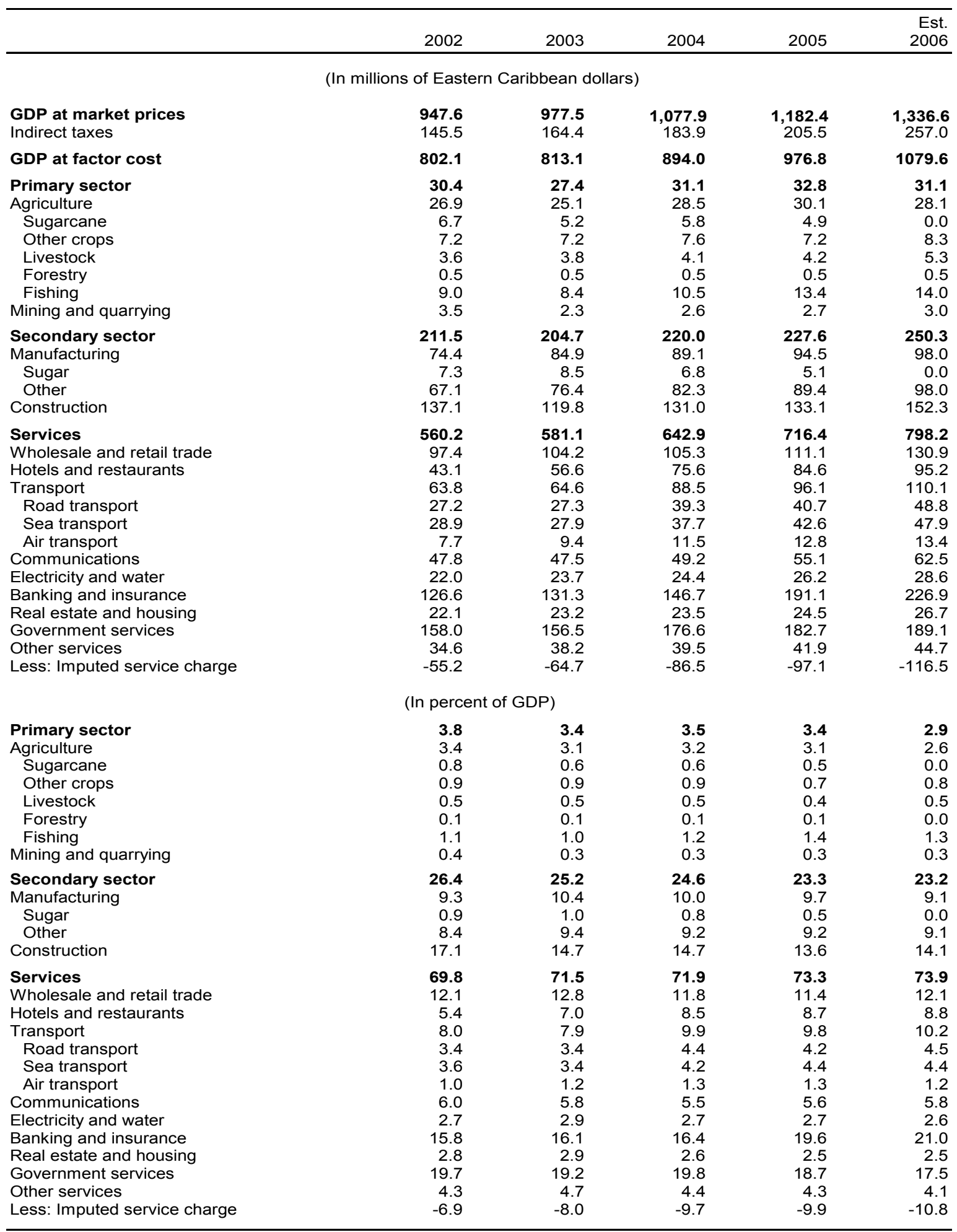

Sources: St. Kitts and Nevis authorities; and Fund staff estimates. 
Table 3. St. Kitts and Nevis: Selected Data on Tourism

\begin{tabular}{|c|c|c|c|c|c|}
\hline & 2002 & 2003 & 2004 & 2005 & $\begin{aligned} \text { Est. } \\
2006 \\
\end{aligned}$ \\
\hline Total number of visitors & 246,364 & 246,788 & 382,290 & 351,105 & 342,741 \\
\hline Cruise ship and yacht & 173,513 & 152,172 & 261,607 & 219,706 & 205,951 \\
\hline Cruise ship & 167,230 & 146,317 & 254,535 & 215,351 & 203,075 \\
\hline Yachts & 6,283 & 5,855 & 7,072 & 4,355 & 2,876 \\
\hline Excursionist & 3,853 & 4,054 & 3,045 & 3,671 & 3,893 \\
\hline Stay-over & 68,998 & 90,562 & 117,638 & 127,728 & 132,897 \\
\hline Hotel and guest houses & 36,600 & 49,808 & 64,703 & 70,250 & 73,072 \\
\hline Other & 32,398 & 40,754 & 52,935 & 57,479 & 59,825 \\
\hline \multicolumn{6}{|l|}{ Stay-over visitors by nationality } \\
\hline United States & 29,577 & 43,418 & 64,859 & 76,526 & 78,645 \\
\hline Canada & 4,538 & 4,617 & 6,325 & 8,233 & 7,605 \\
\hline United Kingdom & 5,622 & 7,982 & 11,004 & 9,536 & 9,909 \\
\hline Caribbean & 27,081 & 31,789 & 31,909 & 27,394 & 32,603 \\
\hline Other & 2,180 & 2,756 & 3,541 & 6,039 & 4,135 \\
\hline \multicolumn{6}{|l|}{ Average period of stay (in days) } \\
\hline All visitors & 3.4 & 4.1 & 3.6 & 4.1 & 4.3 \\
\hline Cruise ship & 1.0 & 1.0 & 1.0 & 1.0 & 1.0 \\
\hline Yachts & 1.0 & 1.0 & 1.0 & 1.0 & 1.0 \\
\hline Excursionist & 1.0 & 1.0 & 1.0 & 1.0 & 1.0 \\
\hline Stay-over & 9.6 & 9.5 & 9.5 & 9.5 & 9.5 \\
\hline Hotel and guest houses & 7.0 & 7.0 & 7.0 & 7.0 & 7.0 \\
\hline Other & 12.5 & 12.5 & 12.5 & 12.5 & 12.5 \\
\hline \multicolumn{6}{|l|}{ Average expenditure per day in E.C. dollars } \\
\hline All visitors & 185.5 & 200.6 & 200.9 & 208.7 & 214.7 \\
\hline Cruise ship & 84.5 & 85.6 & 88.1 & 89.2 & 91.0 \\
\hline Yachts & 84.5 & 85.6 & 88.1 & 89.2 & 91.0 \\
\hline Excursionist & 137.4 & 139.1 & 143.1 & 145.0 & 147.9 \\
\hline Stay-over & 212.3 & 221.3 & 227.6 & 230.5 & 235.1 \\
\hline Hotel and guest houses & 411.0 & 416.4 & 428.2 & 433.8 & 442.4 \\
\hline Other & 86.6 & 87.8 & 90.3 & 91.4 & 93.3 \\
\hline Capacity of rooms in hotels and guest houses & 2,056 & 2,306 & 2,306 & 2,306 & $\ldots$ \\
\hline St. Kitts & 1,637 & 1,887 & 1,887 & 1,887 & $\ldots$ \\
\hline Nevis & 419 & 419 & 419 & 419 & ... \\
\hline \multicolumn{6}{|l|}{ Total expenditure in E.C. dollars } \\
\hline All visitors & 155,587 & 203,473 & 277,133 & 299,123 & 315,362 \\
\hline Cruise ship & 14,136 & 12,529 & 22,414 & 19,210 & 18,477 \\
\hline Yachts & 531 & 501 & 623 & 388 & 262 \\
\hline Excursionist & 529 & 564 & 436 & 532 & 576 \\
\hline Stay-over & 140,391 & 189,879 & 253,660 & 278,992 & 296,047 \\
\hline Hotel and guest houses & 105,304 & 145,168 & 193,936 & 213,299 & 226,304 \\
\hline Other & 35,088 & 44,711 & 59,724 & 65,694 & 69,743 \\
\hline $\begin{array}{l}\text { Total expenditure in thousands of E.C. dollars } \\
\text { Total expenditure in thousands of U.S. dollars }\end{array}$ & $\begin{array}{r}154,258 \\
57,133\end{array}$ & $\begin{array}{r}203,450 \\
75,352\end{array}$ & $\begin{array}{l}277,140 \\
102,644\end{array}$ & $\begin{array}{l}309,150 \\
114,500\end{array}$ & $\begin{array}{l}314,120 \\
116,341\end{array}$ \\
\hline \multicolumn{6}{|c|}{ (Annual percentage changes) } \\
\hline Total expenditure & -7.6 & 31.9 & 36.2 & 11.6 & 1.6 \\
\hline Total visitors & -26.1 & 0.2 & 54.9 & -8.2 & -2.4 \\
\hline Stay-over & -2.2 & 31.3 & 29.9 & 8.6 & 4.0 \\
\hline Cruise ship and yacht & -33.0 & -12.3 & 71.9 & -16.0 & -6.3 \\
\hline Excursionist & -9.8 & -6.8 & 20.8 & -38.4 & -34.0 \\
\hline
\end{tabular}

Sources: St. Kitts and Nevis authorities; Eastern Caribbean Central Bank; and Fund staff estimates. 
Table 4. St. Kitts and Nevis: Selected Indicators of Agricultural Production

\begin{tabular}{lrrrrr}
\hline & 2002 & 2003 & 2004 & 2005 & 2006 \\
\hline & & & & & \\
Tons of cane (in thousands of metric tons) & 227.7 & 169.5 & 169.2 & 142.7 & $\ldots$ \\
Tons of sugar (in thousand of metric tons) & 21.4 & 16.3 & 14.2 & 10.7 & $\ldots$ \\
Area reaped (in thousand of hectares) & 3.5 & 3.2 & 2.8 & 2.1 & $\ldots$ \\
Tons of cane per hectare & 64.4 & 53.4 & 59.8 & 67.8 & $\ldots$ \\
Tons of cane per tons of sugar & 10.6 & 10.4 & 12.0 & 13.3 & $\ldots$ \\
& & & & & \\
Bananas (in tons) & 20.6 & 19.8 & 20.9 & 43.4 & 21.2 \\
Cabbages (in tons) & 52.4 & 91.4 & 101.6 & 66.4 & 69.6 \\
Carrots (in tons) & 106.1 & 107.0 & 88.6 & 77.9 & 82.8 \\
Cucumbers (in tons) & 79.5 & 88.2 & 83.0 & 88.3 & 91.6 \\
Mangoes (in thousand units) & 646.2 & 410.2 & 461.1 & $1,020.4$ & 692.6 \\
Pumpkins (in tons) & 145.9 & 110.0 & 161.3 & 114.6 & 120.5 \\
Sweet oranges (in thousand units) & 30.0 & 20.0 & 21.0 & 21.0 & 21.0 \\
Sweet potatoes (in tons) & 184.4 & 165.9 & 169.0 & 157.9 & 255.3 \\
Tomatoes (in tons) & 133.9 & 180.1 & 158.0 & 107.9 & 172.9 \\
White potatoes (in tons) & 110.7 & 99.8 & 119.7 & 88.0 & 109.8 \\
\hline
\end{tabular}

Sources: St. Kitts and Nevis authorities; and St. Kitts Sugar Manufacturing Corporation. 
Table 5. St. Kitts and Nevis: Average Annual Wage Rate of Workers Registered at the Social Security Board

(In Eastern Caribbean dollars)

\begin{tabular}{lrrrrr}
\hline & 2002 & 2003 & 2004 & 2005 & 2006 \\
\hline & & & & & \\
Age group & & & & & \\
$0-15$ & 1,799 & 1,506 & 1,506 & 1,942 & 1,778 \\
$16-19$ & 6,944 & 6,600 & 6,837 & 7,272 & 7,699 \\
$20-24$ & 13,166 & 13,578 & 14,471 & 15,273 & 15,834 \\
$25-29$ & 17,448 & 18,276 & 19,561 & 20,324 & 21,224 \\
$30-34$ & 21,517 & 22,009 & 24,137 & 24,971 & 25,939 \\
$35-39$ & 22,082 & 23,014 & 25,199 & 26,703 & 28,770 \\
$40-44$ & 23,029 & 23,544 & 26,095 & 26,224 & 28,269 \\
$45-49$ & 25,281 & 24,567 & 26,240 & 27,740 & 29,594 \\
$50-54$ & 27,589 & 28,399 & 30,380 & 30,515 & 31,542 \\
$55-59$ & 25,032 & 26,434 & 28,686 & 29,819 & 30,576 \\
$60-62$ & 22,150 & 23,356 & 24,020 & 26,549 & 30,855 \\
$63-100$ & 16,258 & 16,236 & 20,990 & 21,801 & 23,595 \\
& & & & & \\
Memorandum item: & & & & & \\
Weighted average wage of workers & $\mathbf{1 9 , 8 1 1}$ & $\mathbf{2 0 , 3 0 8}$ & $\mathbf{2 2 , 0 0 1}$ & $\mathbf{2 2 , 8 6 7}$ & $\mathbf{2 4 , 2 1 6}$ \\
\hline
\end{tabular}

Source: Social Security Board. 
Table 6. St. Kitts and Nevis: Number of Workers in the Social Security Scheme by Age Group

\begin{tabular}{lrrrrr}
\hline & 2002 & 2003 & 2004 & 2005 & 2006 \\
& & & & & \\
\hline & & & & & \\
Age group & & & & & \\
$0-15$ & 41 & 42 & 45 & 48 & 1,574 \\
$16-19$ & 1,199 & 1,208 & 1,431 & 1,589 & 3,604 \\
$20-24$ & 3,348 & 3,474 & 3,599 & 3,675 & 3,429 \\
$25-29$ & 3,320 & 3,198 & 3,335 & 3,507 & 3,267 \\
$30-34$ & 3,181 & 3,120 & 3,136 & 3,187 & 3,098 \\
$35-39$ & 3,161 & 3,115 & 3,084 & 3,126 & 2,982 \\
$40-44$ & 2,860 & 2,857 & 2,915 & 3,017 & 2,638 \\
$45-49$ & 2,264 & 2,329 & 2,507 & 2,684 & 1,876 \\
$50-54$ & 1,255 & 1,390 & 1,563 & 1,795 & 959 \\
$55-59$ & 753 & 773 & 826 & 888 & 369 \\
$60-62$ & 309 & 310 & 340 & 357 & 536 \\
$63-100$ & 658 & 638 & 580 & 639 & 24,399 \\
Total number of workers & & & & & \\
\hline
\end{tabular}

Source: Social Security Board. 
Table 7. St. Kitts and Nevis: Retail Price Index

\begin{tabular}{|c|c|c|c|c|c|}
\hline & 2002 & 2003 & 2004 & 2005 & $\begin{array}{r}\text { Est. } \\
2006\end{array}$ \\
\hline \multicolumn{6}{|c|}{ (Index: January 1978=100) } \\
\hline \multicolumn{6}{|l|}{ Period average } \\
\hline All items & 103.0 & 105.3 & 107.7 & 111.4 & 120.8 \\
\hline Food & 102.5 & 103.8 & 106.8 & 110.0 & 119.0 \\
\hline Alcoholic drinks and beverages & 101.9 & 102.8 & 103.2 & 103.9 & 106.7 \\
\hline Clothing and footwear & 105.7 & 107.4 & 107.9 & 109.6 & 115.2 \\
\hline Accommodations & 100.1 & 100.7 & 104.3 & 107.4 & 113.7 \\
\hline Medical services & 121.8 & 131.0 & 131.0 & 131.7 & 143.4 \\
\hline Fuel and light & 100.0 & 100.1 & 100.1 & 101.9 & 104.8 \\
\hline Household supplies and operations & 105.6 & 107.1 & 108.7 & 109.4 & 111.5 \\
\hline Transportation & 100.5 & 105.5 & 109.6 & 119.6 & 141.7 \\
\hline Education & 100.4 & 101.2 & 102.0 & 102.1 & 113.6 \\
\hline Personal services & 100.0 & 103.0 & 103.0 & 103.1 & 108.0 \\
\hline Other services & 100.7 & 102.9 & 104.4 & 105.5 & 109.7 \\
\hline \multicolumn{6}{|l|}{ End of period } \\
\hline All items & 103.6 & 106.6 & 108.4 & 115.0 & 124.1 \\
\hline Food & 102.1 & 106.1 & 108.4 & 114.1 & 121.3 \\
\hline Alcoholic drinks and beverages & 102.6 & 102.8 & 103.7 & 104.4 & 109.1 \\
\hline Clothing and footwear & 106.5 & 106.9 & 107.6 & 111.9 & 121.6 \\
\hline Accommodations & 100.1 & 101.0 & 107.6 & 108.0 & 115.5 \\
\hline Medical services & 130.9 & 131.0 & 131.0 & 134.6 & 149.9 \\
\hline Fuel and light & 100.1 & 100.1 & 100.2 & 105.0 & 104.4 \\
\hline Household supplies and operations & 106.0 & 108.0 & 107.3 & 111.6 & 113.1 \\
\hline Transportation & 100.8 & 108.3 & 110.3 & 127.5 & 146.9 \\
\hline Education & 100.6 & 101.9 & 102.1 & 102.3 & 121.0 \\
\hline Personal services & 100.0 & 103.0 & 103.0 & 103.5 & 109.1 \\
\hline Other services & 101.9 & 104.0 & 104.6 & 109.2 & 114.2 \\
\hline \multicolumn{6}{|c|}{ (Annual percentage change) } \\
\hline \multicolumn{6}{|l|}{ Period average } \\
\hline All items & 2.1 & 2.3 & 2.2 & 3.4 & 8.5 \\
\hline Food & 1.3 & 1.3 & 2.9 & 3.0 & 8.1 \\
\hline Alcoholic drinks and beverages & 0.9 & 0.9 & 0.4 & 0.7 & 2.7 \\
\hline Clothing and footwear & 3.1 & 1.5 & 0.5 & 1.6 & 5.1 \\
\hline Accommodations & 0.1 & 0.6 & 3.6 & 3.0 & 5.8 \\
\hline Medical services & 21.8 & 7.6 & 0.0 & 0.5 & 8.9 \\
\hline Fuel and light & 0.0 & 0.0 & 0.1 & 1.7 & 2.8 \\
\hline Household supplies and operations & 3.2 & 1.5 & 1.5 & 0.6 & 1.9 \\
\hline Transportation & 0.5 & 5.0 & 3.8 & 9.1 & 18.5 \\
\hline Education & 0.3 & 0.8 & 0.8 & 0.1 & 11.3 \\
\hline Personal services & 0.0 & 3.0 & 0.0 & 0.0 & 4.8 \\
\hline Other services & 0.6 & 2.2 & 1.4 & 1.1 & 4.0 \\
\hline \multicolumn{6}{|l|}{ End of period } \\
\hline All items & 1.8 & 2.9 & 1.7 & 6.0 & 7.9 \\
\hline Food & 0.5 & 3.9 & 2.2 & 5.3 & 6.3 \\
\hline Alcoholic drinks and beverages & 0.5 & 0.2 & 0.8 & 0.7 & 4.5 \\
\hline Clothing and footwear & 0.6 & 0.3 & 0.7 & 3.9 & 8.7 \\
\hline Accommodations & 0.1 & 0.9 & 6.5 & 0.4 & 6.9 \\
\hline Medical services & 30.9 & 0.1 & 0.0 & 2.7 & 11.4 \\
\hline Fuel and light & 0.1 & 0.0 & 0.1 & 4.8 & -0.5 \\
\hline Household supplies and operations & 0.9 & 1.9 & -0.7 & 4.0 & 1.4 \\
\hline Transportation & 0.8 & 7.5 & 1.8 & 15.6 & 15.3 \\
\hline Education & 0.3 & 1.4 & 0.1 & 0.3 & 18.3 \\
\hline Personal services & 0.0 & 3.0 & 0.0 & 0.4 & 5.4 \\
\hline Other services & 1.7 & 2.1 & 0.6 & 4.4 & 4.6 \\
\hline
\end{tabular}

Source: St. Kitts and Nevis authorities. 
Table 8. St. Kitts and Nevis: Central Government Operations 1/

\begin{tabular}{|c|c|c|c|c|c|}
\hline & 2002 & 2003 & 2004 & 2005 & $\begin{array}{r}\text { Est. } \\
2006\end{array}$ \\
\hline \multicolumn{6}{|c|}{ (In millions of Eastern Caribbean dollars) } \\
\hline Total revenue and grants & 327.4 & 327.5 & 369.4 & 466.6 & 520.3 \\
\hline Current revenue & 299.1 & 321.4 & 365.3 & 434.4 & 484.2 \\
\hline Tax revenue & 212.1 & 233.9 & 281.9 & 344.8 & 373.1 \\
\hline Nontax revenue & 87.0 & 87.5 & 83.5 & 89.6 & 111.1 \\
\hline Capital revenue and grants & 28.3 & 6.1 & 4.1 & 32.2 & 36.1 \\
\hline Total expenditure and net lending & 511.2 & 415.6 & 454.1 & 514.6 & 591.4 \\
\hline Current expenditure & 326.5 & 333.2 & 376.0 & 435.8 & 480.8 \\
\hline Wages and salaries & 144.2 & 144.1 & 159.7 & 163.1 & 170.0 \\
\hline Goods and services & 78.9 & 79.0 & 95.8 & 114.7 & 127.5 \\
\hline Interest & 69.8 & 74.5 & 76.1 & 95.8 & 128.7 \\
\hline Transfers & 33.6 & 35.6 & 44.3 & 62.2 & 54.7 \\
\hline Net lending & 51.6 & 13.9 & 11.3 & 0.0 & 8.7 \\
\hline Capital expenditure & 133.1 & 68.6 & 66.7 & 78.8 & 101.8 \\
\hline Current balance & -27.4 & -11.7 & -10.7 & -1.4 & 3.4 \\
\hline Overall balance & -183.8 & -88.2 & -84.7 & -48.0 & -71.0 \\
\hline Financing & 113.0 & 113.7 & 114.5 & 132.4 & 138.2 \\
\hline Net foreign financing & 104.3 & 141.8 & 16.6 & -12.6 & 3.0 \\
\hline Drawings & 134.1 & 187.1 & 62.9 & 7.5 & 45.2 \\
\hline Amortization & 26.3 & 42.1 & 45.3 & 55.6 & 49.8 \\
\hline Other & -3.4 & -3.2 & -1.0 & 35.5 & 7.7 \\
\hline Net domestic financing & 2.2 & -30.3 & 93.3 & 141.0 & 128.2 \\
\hline Banking system & -15.0 & -68.5 & 61.8 & 88.7 & 105.7 \\
\hline Nonbanks and other & 17.2 & 38.2 & 31.5 & 52.3 & 22.6 \\
\hline Sale/purchase of assets & 6.5 & 2.2 & 4.6 & 4.0 & 7.0 \\
\hline Statistical discrepancy & 70.9 & -25.6 & -29.8 & -84.4 & -67.2 \\
\hline \multicolumn{6}{|c|}{ (In percent of GDP) } \\
\hline Total revenue and grants & 34.5 & 33.5 & 34.3 & 39.5 & 38.9 \\
\hline Current revenue & 31.6 & 32.9 & 33.9 & 36.7 & 36.2 \\
\hline Tax revenue & 22.4 & 23.9 & 26.2 & 29.2 & 27.9 \\
\hline Nontax revenue & 9.2 & 9.0 & 7.7 & 7.6 & 8.3 \\
\hline Capital revenue and grants & 3.0 & 0.6 & 0.4 & 2.7 & 2.7 \\
\hline Total expenditure and net lending & 53.9 & 42.5 & 42.1 & 43.5 & 44.2 \\
\hline Current expenditure & 34.5 & 34.1 & 34.9 & 36.9 & 36.0 \\
\hline Wages and salaries & 15.2 & 14.7 & 14.8 & 13.8 & 12.7 \\
\hline Goods and services & 8.3 & 8.1 & 8.9 & 9.7 & 9.5 \\
\hline Interest & 7.4 & 7.6 & 7.1 & 8.1 & 9.6 \\
\hline Transfers & 3.5 & 3.6 & 4.1 & 5.3 & 4.1 \\
\hline Net lending & 5.4 & 1.4 & 1.1 & 0.0 & 0.7 \\
\hline Capital expenditure & 14.0 & 7.0 & 6.2 & 6.7 & 7.6 \\
\hline Current balance & -2.9 & -1.2 & -1.0 & -0.1 & 0.3 \\
\hline Overall balance (after grants) & -19.4 & -9.0 & -7.9 & -4.1 & -5.3 \\
\hline Financing & 11.9 & 11.6 & 10.6 & 11.2 & 10.3 \\
\hline Net foreign financing & 11.0 & 14.5 & 1.5 & -1.1 & 0.2 \\
\hline Drawings & 14.1 & 19.1 & 5.8 & 0.6 & 3.4 \\
\hline Amortization & 2.8 & 4.3 & 4.2 & 4.7 & 3.7 \\
\hline Other & -0.4 & -0.3 & -0.1 & 3.0 & 0.6 \\
\hline Net domestic financing & 0.2 & -3.1 & 8.7 & 11.9 & 9.6 \\
\hline Banking system & -1.6 & -7.0 & 5.7 & 7.5 & 7.9 \\
\hline Nonbanks and other & 1.8 & 3.9 & 2.9 & 4.4 & 1.7 \\
\hline Sale/purchase of assets & 0.7 & 0.2 & 0.4 & 0.3 & 0.5 \\
\hline Statistical discrepancy & 7.5 & -2.6 & -2.8 & -7.1 & -5.0 \\
\hline
\end{tabular}

Sources: Ministry of Finance; and Fund staff estimates.

1/ Combined accounts of the St. Kitts Government and the Nevis Island Administration. 
Table 9. St. Kitts and Nevis: Central Government Revenue 1/

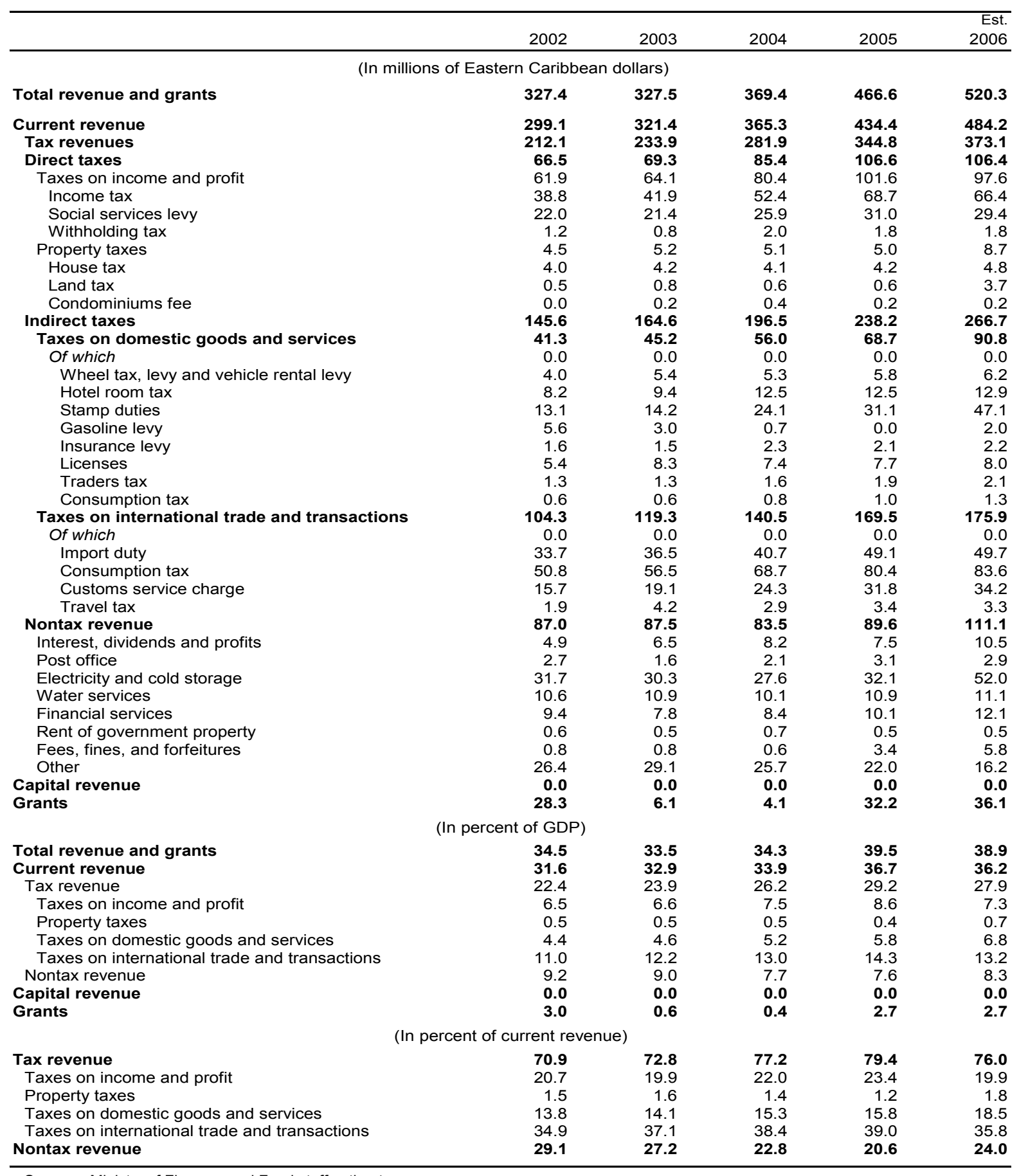

Sources: Ministry of Finance; and Fund staff estimates.

1/ Combined accounts of the St. Kitts Government and the Nevis Island Administration. 
Table 10. St. Kitts and Nevis: Central Government Expenditure 1/

\begin{tabular}{|c|c|c|c|c|c|}
\hline & 2002 & 2003 & 2004 & 2005 & $\begin{array}{r}\text { Est. } \\
2006\end{array}$ \\
\hline \multicolumn{6}{|c|}{ (In millions of Eastern Caribbean dollars) } \\
\hline Total expenditure and net lending & 511.2 & 415.6 & 454.1 & 514.6 & 591.4 \\
\hline Current expenditure & 326.5 & 333.2 & 376.0 & 435.8 & 480.8 \\
\hline Wages and salaries & 144.2 & 144.1 & 159.7 & 163.1 & 170.0 \\
\hline Goods and services & 78.9 & 79.0 & 95.8 & 114.7 & 127.5 \\
\hline Interest & 69.8 & 74.5 & 76.1 & 95.8 & 128.7 \\
\hline Domestic & 38.2 & 31.0 & 30.1 & 48.4 & 87.0 \\
\hline Foreign & 31.6 & 43.5 & 46.1 & 47.4 & 41.7 \\
\hline Transfers & 33.6 & 35.6 & 44.3 & 62.2 & 54.7 \\
\hline Net lending & 51.6 & 13.9 & 11.3 & 0.0 & 8.7 \\
\hline Capital expenditure & 133.1 & 68.6 & 66.7 & 78.8 & 101.8 \\
\hline \multicolumn{6}{|c|}{ (In percent of GDP) } \\
\hline Total expenditure and net lending & 53.9 & 42.5 & 42.1 & 43.5 & 44.2 \\
\hline Current expenditure & 34.5 & 34.1 & 34.9 & 36.9 & 36.0 \\
\hline Wages and salaries & 15.2 & 14.7 & 14.8 & 13.8 & 12.7 \\
\hline Goods and services & 8.3 & 8.1 & 8.9 & 9.7 & 9.5 \\
\hline Interest & 7.4 & 7.6 & 7.1 & 8.1 & 9.6 \\
\hline Domestic & 4.0 & 3.2 & 2.8 & 4.1 & 6.5 \\
\hline Foreign & 3.3 & 4.5 & 4.3 & 4.0 & 3.1 \\
\hline Transfers & 3.5 & 3.6 & 4.1 & 5.3 & 4.1 \\
\hline Net lending & 5.4 & 1.4 & 1.1 & 0.0 & 0.7 \\
\hline Capital expenditure & 14.0 & 7.0 & 6.2 & 6.7 & 7.6 \\
\hline
\end{tabular}

Sources: Ministry of Finance; and Fund staff estimates.

1/ Combined accounts of the St. Kitts Government and the Nevis Island Administration. 
Table 11. St. Kitts and Nevis: Central Government Domestic Debt 1/

\begin{tabular}{|c|c|c|c|c|c|}
\hline & 2002 & 2003 & 2004 & 2005 & $\begin{array}{r}\text { Est. } \\
2006\end{array}$ \\
\hline \multicolumn{6}{|c|}{ (In millions of Eastern Caribbean dollars) } \\
\hline Treasury bills & 256.9 & 301.3 & 352.1 & 411.4 & 388.8 \\
\hline ECCB & 0.0 & 5.8 & 5.8 & 5.8 & 5.8 \\
\hline Commercial banks & 158.9 & 180.7 & 206.6 & 234.9 & 216.7 \\
\hline Nonbanks & 98.0 & 114.8 & 139.7 & 170.7 & 166.3 \\
\hline \multicolumn{6}{|l|}{ Of which } \\
\hline Social Security & 22.4 & 23.6 & 27.3 & 27.3 & 27.3 \\
\hline Debentures & 28.9 & 27.6 & 27.8 & 24.3 & 28.0 \\
\hline ECCB & 3.5 & 3.5 & 3.5 & 3.5 & 7.2 \\
\hline Commercial banks & 6.2 & 6.2 & 3.5 & 3.5 & 3.5 \\
\hline Nonbanks & 19.2 & 17.9 & 20.8 & 17.3 & 17.3 \\
\hline \multicolumn{6}{|l|}{ Of which } \\
\hline Social Security & 16.5 & 16.5 & 16.5 & 16.5 & 16.5 \\
\hline Loans and advances & 173.4 & 119.6 & 188.0 & 279.4 & 417.0 \\
\hline ECCB & 3.6 & 0.7 & 3.7 & 3.7 & 0.6 \\
\hline Commercial banks & 117.5 & 69.2 & 130.3 & 225.3 & 351.8 \\
\hline Social Security & 45.6 & 44.2 & 42.8 & 41.4 & 40.1 \\
\hline Other & 6.8 & 5.5 & 11.1 & 9.0 & 24.5 \\
\hline Regional Government Securities Market (RGSM) & 75.0 & 75.0 & 75.0 & 42.1 & 42.1 \\
\hline Other & 0.0 & 0.0 & 0.0 & 32.7 & 28.6 \\
\hline Domestic debt stock & 534.2 & 523.5 & 642.9 & 789.9 & 904.5 \\
\hline \multicolumn{6}{|l|}{ Memorandum items: } \\
\hline Deposits with ECCB & 5.1 & 15.6 & 7.1 & 6.9 & 6.4 \\
\hline Deposits with commercial banks & 14.8 & 23.8 & 58.5 & 55.7 & 95.3 \\
\hline Average interest rate on domestic debt (in percent) & 7.7 & 5.9 & 5.2 & 6.8 & 8.0 \\
\hline External debt & 494.8 & 643.6 & 663.2 & 596.1 & 600.6 \\
\hline \multicolumn{6}{|c|}{ (In percent of domestic debt) } \\
\hline Total domestic debt & 100.0 & 100.0 & 100.0 & 100.0 & 100.0 \\
\hline Treasury bills & 48.1 & 57.6 & 54.8 & 52.1 & 43.0 \\
\hline Debentures & 5.4 & 5.3 & 4.3 & 3.1 & 3.1 \\
\hline Loans and advances & 32.5 & 22.8 & 29.2 & 35.4 & 46.1 \\
\hline RGSM & 14.0 & 14.3 & 11.7 & 5.3 & 4.7 \\
\hline Other & 0.0 & 0.0 & 0.0 & 4.1 & 3.2 \\
\hline \multicolumn{6}{|c|}{ (In percent of GDP) } \\
\hline Total debt & 108.6 & 119.4 & 121.2 & 117.2 & 112.6 \\
\hline Domestic debt & 56.4 & 53.6 & 59.6 & 66.8 & 67.7 \\
\hline External debt & 52.2 & 65.8 & 61.5 & 50.4 & 44.9 \\
\hline \multicolumn{6}{|c|}{ (In percent of total debt) } \\
\hline Domestic debt & 51.9 & 44.9 & 49.2 & 57.0 & 60.1 \\
\hline External debt & 48.1 & 55.1 & 50.8 & 43.0 & 39.9 \\
\hline
\end{tabular}

Sources: Ministry of Finance; Eastern Caribbean Central Bank; and Fund staff estimates.

1/ Combined debt of the St. Kitts Government and the Nevis Island Administration. Does not include debt of public enterprises. 
Table 12. St. Kitts and Nevis: Operations of the Social Security Board

\begin{tabular}{|c|c|c|c|c|c|}
\hline & 2002 & 2003 & 2004 & 2005 & 2006 \\
\hline \multicolumn{6}{|c|}{ (In millions of Eastern Caribbean dollars) } \\
\hline Total revenue & 79.6 & 84.1 & 94.6 & 101.4 & 109.0 \\
\hline Contributions & 46.9 & 48.0 & 53.8 & 58.1 & 61.0 \\
\hline Investment income & 31.5 & 35.4 & 40.0 & 42.7 & 47.5 \\
\hline Other income & 1.2 & 0.6 & 0.7 & 0.6 & 0.5 \\
\hline Total expenditure & 28.5 & 28.1 & 30.6 & 33.7 & 36.6 \\
\hline Current expenditure & 25.2 & 27.6 & 29.8 & 32.6 & 35.6 \\
\hline Benefits & 18.6 & 20.4 & 22.1 & 24.9 & 27.2 \\
\hline Other & 6.6 & 7.1 & 7.7 & 7.7 & 8.4 \\
\hline Capital expenditure & 3.3 & 0.5 & 0.8 & 1.1 & 1.1 \\
\hline Current balance & 54.4 & 56.5 & 64.8 & 68.7 & 73.4 \\
\hline Overall balance & 51.1 & 56.0 & 64.0 & 67.7 & 72.4 \\
\hline Financing & -51.1 & -56.0 & -64.0 & -67.7 & -72.4 \\
\hline \multicolumn{6}{|l|}{ Of which } \\
\hline Domestic banking system & -2.6 & 13.4 & -44.9 & -59.7 & -39.2 \\
\hline \multicolumn{6}{|c|}{ (In percent of GDP) } \\
\hline Total revenue & 8.4 & 8.6 & 8.8 & 8.6 & 8.2 \\
\hline Total expenditure & 3.0 & 2.9 & 2.8 & 2.9 & 2.7 \\
\hline Current balance & 5.7 & 5.8 & 6.0 & 5.8 & 5.5 \\
\hline Overall balance & 5.4 & 5.7 & 5.9 & 5.7 & 5.4 \\
\hline
\end{tabular}

Source: Social Security Board. 
Table 13. St. Kitts and Nevis: Structure of the Public Debt 1/

(In millions of U.S. dollars, unless otherwise stated)

\begin{tabular}{|c|c|c|c|c|c|}
\hline & 2002 & 2003 & 2004 & 2005 & $\begin{array}{r}\text { Est } \\
2006\end{array}$ \\
\hline Total stock of debt & 571.9 & 656.9 & 778.4 & 853.0 & 913.8 \\
\hline (In percent of GDP) & 163.0 & 181.5 & 195.0 & 194.8 & 184.6 \\
\hline Total external debt (by debtor) & 266.4 & 320.6 & 341.5 & 337.3 & 331.0 \\
\hline St. Kitts Government & 138.9 & 185.5 & 180.7 & 173.8 & 178.3 \\
\hline Nevis Island Administration & 44.4 & 52.9 & 65.0 & 47.0 & 44.1 \\
\hline Public enterprises & 83.1 & 82.2 & 95.9 & 116.5 & 108.6 \\
\hline Total external debt (by creditor) & 266.4 & 320.6 & 341.5 & 337.3 & 331.0 \\
\hline Bilateral & 41.2 & 47.7 & 42.7 & 36.4 & 41.2 \\
\hline Multilateral & 84.3 & 91.7 & 97.6 & 98.9 & 105.6 \\
\hline Commercial and other & 140.8 & 181.2 & 201.2 & 202.0 & 184.2 \\
\hline Total domestic debt (by debtor) & 305.5 & 336.4 & 436.8 & 515.8 & 582.8 \\
\hline St. Kitts Government & 178.0 & 168.0 & 207.8 & 255.9 & 289.8 \\
\hline Nevis Island Administration & 19.8 & 25.9 & 30.3 & 36.6 & 45.2 \\
\hline Public enterprises & 107.7 & 142.5 & 198.7 & 223.2 & 247.8 \\
\hline Total domestic debt (by instruments) & 305.5 & 336.4 & 436.8 & 515.8 & 582.8 \\
\hline Treasury bills & 95.1 & 111.6 & 130.4 & 152.4 & 144.0 \\
\hline Debentures & 10.7 & 10.2 & 10.3 & 9.0 & 10.4 \\
\hline Loans and advances & 171.9 & 186.8 & 268.3 & 326.7 & 402.2 \\
\hline Regional Government Securities Market & 27.8 & 27.8 & 27.8 & 15.6 & 15.6 \\
\hline Other & 0.0 & 0.0 & 0.0 & 12.1 & 10.6 \\
\hline \multicolumn{6}{|l|}{ Memorandum item (in percent of GDP): } \\
\hline Net assets of Social Security Board & 58.6 & 64.0 & 63.8 & 64.1 & 62.3 \\
\hline
\end{tabular}

Sources: St. Kitts and Nevis authorities; Eastern Caribbean Central Bank; and Fund staff estimates.

1/ Public debt comprises all debts of the St. Kitts Government, Nevis Island Administration, and public enterprises, including government guaranteed debt. 
Table 14. St. Kitts and Nevis: Monetary Survey

\begin{tabular}{|c|c|c|c|c|c|}
\hline & 2002 & 2003 & 2004 & 2005 & $\begin{array}{r}\text { Est. } \\
2006\end{array}$ \\
\hline \multicolumn{6}{|c|}{ (In millions of Eastern Caribbean dollars) } \\
\hline Net foreign assets & 324.0 & 438.9 & 387.6 & 480.7 & 562.1 \\
\hline ECCB imputed net international reserves & 176.6 & 174.6 & 211.5 & 193.0 & 239.1 \\
\hline Crown agents & 9.5 & 9.6 & 10.6 & 10.6 & 10.6 \\
\hline Commercial banks & 137.9 & 254.7 & 165.5 & 277.0 & 312.3 \\
\hline Net domestic assets & 520.8 & 464.1 & 715.2 & 670.2 & 736.2 \\
\hline Net credit to the public sector & 143.0 & 51.4 & 216.4 & 318.0 & 392.1 \\
\hline Net credit to central government & 266.7 & 198.2 & 263.0 & 351.7 & 422.5 \\
\hline Net credit to social security & -331.1 & -317.7 & -362.6 & -422.3 & -461.5 \\
\hline Net credit to public enterprises & 207.5 & 170.9 & 316.1 & 388.6 & 431.1 \\
\hline Net credit to nonbank financial institutions & -16.0 & 25.1 & 48.8 & 41.0 & 37.9 \\
\hline Credit to the private sector & 658.9 & 687.4 & 748.5 & 804.6 & 899.5 \\
\hline Net other assets & -265.1 & -299.8 & -298.5 & -493.4 & -593.3 \\
\hline Broad money & 844.8 & 903.0 & 1102.9 & 1150.8 & 1298.3 \\
\hline Money & 119.9 & 133.1 & 166.9 & 163.7 & 184.5 \\
\hline Currency in circulation & 37.7 & 39.9 & 44.6 & 49.9 & 55.1 \\
\hline Demand deposits & 82.2 & 93.2 & 122.3 & 113.8 & 129.4 \\
\hline Quasi-money & 724.9 & 769.9 & 936.0 & 987.1 & 1113.8 \\
\hline Savings deposits & 335.0 & 361.2 & 418.2 & 468.1 & 518.9 \\
\hline Time deposits & 153.6 & 144.6 & 203.5 & 183.3 & 188.1 \\
\hline Foreign currency deposits & 236.3 & 264.2 & 314.2 & 335.7 & 406.8 \\
\hline \multicolumn{6}{|c|}{ (Changes in percent of broad money at beginning of period) } \\
\hline Net foreign assets & 10.2 & 13.6 & -5.7 & 8.4 & 7.1 \\
\hline Net domestic assets & -3.9 & -6.7 & 27.8 & -4.1 & 5.7 \\
\hline Net credit to the public sector & 1.6 & -10.8 & 18.3 & 9.2 & 6.4 \\
\hline Net credit to central government & -2.5 & -8.1 & 7.2 & 8.0 & 6.2 \\
\hline Net credit to public enterprises & 4.0 & -2.7 & 11.1 & 1.2 & 0.3 \\
\hline Net credit to nonbank financial institutions & -1.4 & 4.9 & 2.6 & -0.7 & -0.3 \\
\hline Credit to the private sector & -2.1 & 3.4 & 6.8 & 5.1 & 8.2 \\
\hline Other assets, net $1 /$ & -1.9 & -4.1 & 0.1 & -17.7 & -8.7 \\
\hline \multicolumn{6}{|c|}{ (Annual percentage changes, unless otherwise specified) } \\
\hline Broad money & 6.4 & 6.9 & 22.1 & 4.3 & 12.8 \\
\hline Money & 12.9 & 11.0 & 25.4 & -1.9 & 12.7 \\
\hline Currency in circulation & 4.4 & 5.9 & 11.7 & 11.8 & 10.5 \\
\hline Demand deposits & 17.3 & 13.4 & 31.2 & -6.9 & 13.7 \\
\hline Quasi-money & 5.4 & 6.2 & 21.6 & 5.5 & 12.8 \\
\hline Credit to the private sector & -2.4 & 4.3 & 8.9 & 7.5 & 11.8 \\
\hline \multicolumn{6}{|l|}{ Memorandum items: } \\
\hline Income velocity of money & 7.9 & 7.3 & 6.5 & 7.2 & 7.2 \\
\hline Income velocity of broad money & 1.1 & 1.1 & 1.0 & 1.0 & 1.0 \\
\hline Weighted average deposit interest rate (in percent per annum) $2 /$ & 3.8 & 4.1 & 3.6 & 4.1 & 3.7 \\
\hline Weighted average lending interest rate (in percent per annum) $2 /$ & 10.4 & 12.0 & 9.9 & 9.7 & 9.4 \\
\hline
\end{tabular}

Sources: Eastern Caribbean Central Bank; and Fund staff estimates.

$1 /$ Includes subsidiaries and affiliates and capital accounts.

2/ End of period. Weighted by size of loans or deposits. There was a break in the average interest rate series in June 2003. 
Table 15. St. Kitts and Nevis: Eastern Caribbean Central Bank Local Operations (In millions of Eastern Caribbean dollars, end of period)

\begin{tabular}{|c|c|c|c|c|c|}
\hline & 2002 & 2003 & 2004 & 2005 & $\begin{aligned} \text { Est. } \\
2006\end{aligned}$ \\
\hline Net imputed international reserves & 176.6 & 174.6 & 211.5 & 193.0 & 239.1 \\
\hline Net claims on commercial banks & -130.3 & -109.1 & -158.2 & -143.8 & -182.5 \\
\hline Claims & 9.6 & 13.9 & 6.7 & 0.4 & 0.9 \\
\hline Loans & 9.6 & 13.9 & 6.7 & 0.4 & 0.9 \\
\hline Deposits with banks & 0.0 & 0.0 & 0.0 & 0.0 & 0.0 \\
\hline Liabilities & 139.9 & 123.0 & 164.9 & 144.2 & 183.4 \\
\hline Currency & 14.8 & 16.7 & 16.0 & 15.9 & 23.2 \\
\hline Current account & 123.3 & 105.6 & 148.3 & 126.3 & 159.8 \\
\hline Current deposits & 0.0 & 0.0 & 0.0 & 0.0 & 0.0 \\
\hline Fixed deposits & 1.7 & 0.7 & 0.6 & 2.0 & 0.4 \\
\hline Net credit to central government & 2.0 & -11.4 & 0.2 & 0.4 & 1.5 \\
\hline Treasury bills & 0.0 & 0.0 & 0.0 & 0.0 & 0.0 \\
\hline Debentures & 3.5 & 3.5 & 3.5 & 3.5 & 7.2 \\
\hline Temporary advances & 3.0 & 0.7 & 3.7 & 3.7 & 0.6 \\
\hline Other claims & 0.5 & 0.0 & 0.0 & 0.0 & 0.0 \\
\hline Central government deposits (-) & -5.1 & -15.6 & -7.1 & -6.9 & -6.4 \\
\hline Net other assets & -10.6 & -14.2 & -8.9 & 0.3 & -3.0 \\
\hline Liabilities to the private sector & 37.7 & 39.9 & 44.6 & 49.9 & 55.1 \\
\hline Currency in circulation & 52.5 & 56.6 & 60.6 & 65.8 & 78.3 \\
\hline Currency held by banks & -14.8 & -16.7 & -16.0 & -15.9 & -23.2 \\
\hline \multicolumn{6}{|l|}{ Memorandum items: } \\
\hline \multicolumn{6}{|l|}{ St. Kitts and Nevis imputed net international reserves to } \\
\hline $\begin{array}{l}\text { ECCU total net international reserves (in percent) 1/ } \\
\text { St. Kitts and Nevis reserve money to }\end{array}$ & 13.0 & 12.1 & 12.4 & 12.0 & 12.8 \\
\hline ECCU total reserve money (in percent) $1 /$ & 13.4 & 13.3 & 12.5 & 12.7 & 13.4 \\
\hline
\end{tabular}

Sources: Eastern Caribbean Central Bank; and Fund staff estimates.

1/ The ECCU member territories are Anguilla, Antigua and Barbuda, Dominica, Grenada, Montserrat, St. Kitts and Nevis, St. Lucia, and St. Vincent and the Grenadines. 
Table 16. St. Kitts and Nevis: Consolidated Balance Sheet Accounts of the Commercial Banks (In millions of Eastern Caribbean dollars)

\begin{tabular}{|c|c|c|c|c|c|}
\hline & 2002 & 2003 & 2004 & 2005 & $\begin{array}{r}\text { Est. } \\
2006\end{array}$ \\
\hline Net foreign assets & 137.9 & 254.7 & 165.5 & 277.0 & 312.3 \\
\hline Foreign currency holdings & 4.3 & 7.9 & 5.6 & 4.2 & 7.8 \\
\hline Claims on ECCB area banks & 62.3 & 12.3 & 60.1 & 204.8 & 241.1 \\
\hline Claims on banks abroad & 328.9 & 531.2 & 428.0 & 387.0 & 294.9 \\
\hline Balances due to ECCB area banks & 195.1 & 249.8 & 247.6 & 252.7 & 243.5 \\
\hline Balances due to banks abroad & 134.9 & 151.8 & 100.1 & 138.0 & 202.0 \\
\hline Nonresident deposits & 130.2 & 123.5 & 146.3 & 181.3 & 157.6 \\
\hline Demand & 17.0 & 16.5 & 18.2 & 28.9 & 21.1 \\
\hline Savings & 40.5 & 39.9 & 59.1 & 68.4 & 79.2 \\
\hline Net position with ECCB & 130.3 & 109.1 & 158.2 & 143.8 & 182.5 \\
\hline Claims on ECCB & 139.9 & 123.0 & 164.9 & 144.2 & 183.4 \\
\hline Currency holdings & 14.8 & 16.7 & 16.0 & 15.9 & 23.2 \\
\hline Current deposits & 123.3 & 105.6 & 148.3 & 126.3 & 159.8 \\
\hline Fixed deposits & 1.7 & 0.7 & 0.6 & 2.0 & 0.4 \\
\hline Liabilities to ECCB & 9.6 & 13.9 & 6.7 & 0.4 & 0.9 \\
\hline Net domestic assets & 546.3 & 506.7 & 741.9 & 688.3 & 756.6 \\
\hline Public sector & 142.1 & 63.2 & 216.3 & 317.6 & 390.7 \\
\hline St. Kitts Government (net) & 224.2 & 171.5 & 215.8 & 296.3 & 396.1 \\
\hline Treasury bills & 155.8 & 158.0 & 171.2 & 161.6 & 146.5 \\
\hline Other securities & 6.2 & 6.2 & 6.2 & 3.5 & 3.5 \\
\hline Loans and investments & 228.5 & 252.1 & 367.4 & 418.4 & 464.8 \\
\hline Deposits & 20.0 & 80.9 & 51.3 & 29.8 & 33.7 \\
\hline Other nonbank financial institutions (net) & -16.0 & 25.1 & 48.8 & 41.0 & 37.9 \\
\hline Loans and investments & 3.8 & 42.0 & 74.4 & 86.9 & 101.0 \\
\hline Deposits & 19.8 & 16.9 & 25.6 & 45.9 & 63.1 \\
\hline Subsidiaries and affiliates & -30.2 & -18.9 & -21.7 & -31.3 & -42.0 \\
\hline Loans and investments & 20.3 & 43.3 & 48.9 & 49.6 & 48.4 \\
\hline Deposits & 50.5 & 62.2 & 70.6 & 80.9 & 90.4 \\
\hline Credit to private sector & 658.9 & 687.4 & 748.5 & 804.6 & 899.5 \\
\hline Interbank float & 1.2 & 6.6 & -5.1 & 27.0 & 3.3 \\
\hline Claims & 9.2 & 28.3 & 7.6 & 44.5 & 25.1 \\
\hline Liabilities & 8.0 & 21.7 & 12.7 & 17.5 & 21.8 \\
\hline Net unclassified assets & -209.7 & -256.7 & -244.9 & -470.7 & -532.8 \\
\hline Assets & 17.4 & 38.7 & 162.2 & 77.3 & 68.6 \\
\hline Liabilities & 227.1 & 295.4 & 407.2 & 548.0 & 601.4 \\
\hline Long-term foreign liabilities & 7.3 & 7.3 & 7.3 & 8.2 & 8.2 \\
\hline Liabilities to the private sector & 807.1 & 863.1 & $1,058.3$ & $1,101.0$ & $1,243.2$ \\
\hline
\end{tabular}

Source: Eastern Caribbean Central Bank. 
Table 17. St. Kitts and Nevis: Sectoral Distribution of Commercial Bank Loans

\begin{tabular}{|c|c|c|c|c|c|}
\hline & 2002 & 2003 & 2004 & 2005 & $\begin{array}{r}\text { Est. } \\
2006\end{array}$ \\
\hline \multicolumn{6}{|c|}{ (In millions of Eastern Caribbean dollars) } \\
\hline Total 1/ & $1,107.2$ & $1,066.8$ & $1,330.1$ & $1,541.1$ & $1,825.3$ \\
\hline Agriculture & 217.0 & 221.3 & 248.6 & 275.2 & 271.4 \\
\hline Fisheries & 0.9 & 0.4 & 0.3 & 0.2 & 0.2 \\
\hline Mining and quarrying & 0.9 & 0.6 & 0.9 & 1.0 & 1.0 \\
\hline Manufacturing & 20.2 & 19.3 & 19.1 & 17.4 & 22.8 \\
\hline Food and beverages & 8.3 & 7.6 & 8.0 & 7.7 & 15.0 \\
\hline Clothing & 2.6 & 2.1 & 2.0 & 1.8 & 1.0 \\
\hline Building materials and metals & 3.9 & 4.5 & 4.1 & 4.0 & 2.7 \\
\hline Other & 5.4 & 5.1 & 4.9 & 3.9 & 4.2 \\
\hline Distributive trade & 121.1 & 109.4 & 123.0 & 128.4 & 129.0 \\
\hline Tourism & 56.4 & 52.5 & 69.4 & 69.0 & 67.4 \\
\hline Entertainment & 4.7 & 4.5 & 7.5 & 6.8 & 7.4 \\
\hline Transport and storage & 5.3 & 6.7 & 7.2 & 7.9 & 10.2 \\
\hline Public utilities & 19.9 & 26.5 & 34.7 & 70.0 & 138.5 \\
\hline Construction and land development & 42.4 & 31.7 & 86.9 & 115.6 & 142.4 \\
\hline Public administration & 154.3 & 70.2 & 158.4 & 225.3 & 350.7 \\
\hline Professional and other services & 40.5 & 40.3 & 36.2 & 39.8 & 39.0 \\
\hline Financial institutions & 6.8 & 10.3 & 31.1 & 28.5 & 27.4 \\
\hline Personal & 416.9 & 473.2 & 506.8 & 556.0 & 617.7 \\
\hline Acquisition of property & 239.0 & 270.9 & 248.0 & 300.5 & 344.6 \\
\hline Durable consumer goods & 15.9 & 16.0 & 19.3 & 26.4 & 29.0 \\
\hline Other & 162.0 & 186.3 & 239.5 & 229.0 & 244.1 \\
\hline \multicolumn{6}{|c|}{ (In percent of total) } \\
\hline Total 1/ & 100.0 & 100.0 & 100.0 & 100.0 & 100.0 \\
\hline Agriculture & 19.6 & 20.7 & 18.7 & 17.9 & 17.6 \\
\hline Fisheries & 0.1 & 0.0 & 0.0 & 0.0 & 0.0 \\
\hline Mining and quarrying & 0.1 & 0.1 & 0.1 & 0.1 & 0.1 \\
\hline Manufacturing & 1.8 & 1.8 & 1.4 & 1.1 & 1.5 \\
\hline Food and beverages & 0.7 & 0.7 & 0.6 & 0.5 & 1.0 \\
\hline Clothing & 0.2 & 0.2 & 0.2 & 0.1 & 0.1 \\
\hline Building materials and metals & 0.4 & 0.4 & 0.3 & 0.3 & 0.2 \\
\hline Other & 0.5 & 0.5 & 0.4 & 0.3 & 0.3 \\
\hline Distributive trade & 10.9 & 10.3 & 9.2 & 8.3 & 8.4 \\
\hline Tourism & 5.1 & 4.9 & 5.2 & 4.5 & 4.4 \\
\hline Entertainment & 0.4 & 0.4 & 0.6 & 0.4 & 0.5 \\
\hline Transport and storage & 0.5 & 0.6 & 0.5 & 0.5 & 0.7 \\
\hline Public utilities & 1.8 & 2.5 & 2.6 & 4.5 & 9.0 \\
\hline Construction and land development & 3.8 & 3.0 & 6.5 & 7.5 & 9.2 \\
\hline Public administration & 13.9 & 6.6 & 11.9 & 14.6 & 22.8 \\
\hline Professional and other services & 3.7 & 3.8 & 2.7 & 2.6 & 2.5 \\
\hline Financial institutions & 0.6 & 1.0 & 2.3 & 1.9 & 1.8 \\
\hline Personal & 37.7 & 44.4 & 38.1 & 36.1 & 40.1 \\
\hline Acquisition of property & 21.6 & 25.4 & 18.6 & 19.5 & 22.4 \\
\hline Durable consumer goods & 1.4 & 1.5 & 1.4 & 1.7 & 1.9 \\
\hline Other & 14.6 & 17.5 & 18.0 & 14.9 & 15.8 \\
\hline
\end{tabular}

Source: Eastern Caribbean Central Bank.

1/ Public and private sector credit. 
Table 18. St. Kitts and Nevis: Selected Interest Rates

(In percent per year, end of period)

\begin{tabular}{|c|c|c|c|c|c|}
\hline & 2002 & 2003 & 2004 & 2005 & 2006 \\
\hline \multicolumn{6}{|l|}{ Deposits } \\
\hline Demand & $1.0-4.0$ & $1.0-4.0$ & $1.0-4.0$ & $0-4.0$ & $0-4.0$ \\
\hline Savings & $3.0-5.5$ & $3.0-5.5$ & $3.0-5.5$ & $3.0-5.5$ & $3.0-5.5$ \\
\hline \multicolumn{6}{|l|}{ Time } \\
\hline Up to 3 months & $3.0-6.0$ & $3.0-6.0$ & $1.0-4.75$ & $1.0-4.75$ & $1.0-4.75$ \\
\hline Over 3 months to 6 months & $3.0-6.0$ & $3.0-6.0$ & $1.0-5.5$ & $1.0-5.5$ & $1.0-5.5$ \\
\hline Over 6 months to 12 months & $2.5-7.0$ & $1.0-7.0$ & $1.0-6.5$ & $1.0-6.5$ & $1.0-6.5$ \\
\hline Over 1 year to 2 years & $2.5-5.0$ & $1.0-7.0$ & $1.0-6.5$ & $1.0-6.5$ & $1.0-6.5$ \\
\hline Over 2 years & $3.75-5$ & $3.25-7.0$ & $1.0-6.5$ & $1.0-6.5$ & $1.0-6.5$ \\
\hline Average deposit interest rate $1 /$ & 3.8 & 4.1 & 3.6 & 4.1 & 3.7 \\
\hline \multicolumn{6}{|l|}{ Lending } \\
\hline Prime & $8.5-11.0$ & $8.5-11.0$ & $8.5-9$ & $8.5-9$ & $8.5-9.0$ \\
\hline Other & $8.5-15.0$ & $8.5-15.0$ & $7.5-21.0$ & $7.5-21.0$ & $7.5-21.0$ \\
\hline \multicolumn{6}{|l|}{ Add-on-loans } \\
\hline Nominal interest rate & $8.0-10.0$ & $8.0-10.0$ & $8.0-10.0$ & $9.0-10.0$ & $9.0-10.0$ \\
\hline Effective interest rate & $11.3-19.0$ & $11.3-19.9$ & $11.3-22.0$ & $14.0-22.0$ & $14.0-22.0$ \\
\hline Average lending interest rate $1 /$ & 10.4 & 12.0 & 9.9 & 9.7 & 9.4 \\
\hline \multicolumn{6}{|l|}{ Government } \\
\hline Treasury bills $2 /$ & 7.5 & 7.2 & 6.5 & 6.5 & 6.5 \\
\hline \multicolumn{6}{|l|}{ Memorandum item: } \\
\hline U.S. dollar certificate of deposit rate $3 /$ & 1.7 & 1.2 & 1.6 & 3.5 & 5.2 \\
\hline
\end{tabular}

Sources: St. Kitts and Nevis authorities; Eastern Caribbean Central Bank; and International Finance Statistics.

1/ There was a break in the average interest rate series in June 2003.

2/ End-month yield on three-month treasury bills.

3/ Three-month CDs. 
Table 19. St. Kitts and Nevis: Selected Performance Indicators of the Commercial Banks (In percent)

\begin{tabular}{|c|c|c|c|c|c|}
\hline & 2002 & 2003 & 2004 & 2005 & 2006 \\
\hline Total capital/risk weighted assets $1 /$ & 44.2 & 33.7 & 33.8 & 45.1 & 38.9 \\
\hline Tier 1 capital/risk weighted assets $1 /$ & 40.0 & 36.6 & 30.0 & 39.7 & 35.0 \\
\hline Total capital/total assets $1 /$ & 14.8 & 11.8 & 11.7 & 12.7 & 13.2 \\
\hline Unsatisfactory assets net of provisions/total capital 2/ & 57.1 & 47.8 & 40.5 & 28.1 & 21.7 \\
\hline Gross government exposure/total capital $1 /$ & 261 & 254 & 323 & 341 & 337 \\
\hline Net government exposure/total capital $1 /$ & 68 & 18 & 87 & 123 & 145 \\
\hline \multicolumn{6}{|l|}{ Asset quality } \\
\hline $\begin{array}{l}\text { Unsatisfactory assets/total gross loans 2/ } \\
\text { Of which }\end{array}$ & \multicolumn{5}{|c|}{ Of which } \\
\hline Locally incorporated banks & 19.8 & 15.6 & 11.2 & 7.9 & 6.6 \\
\hline Gross public sector loans/total gross loans & 31.6 & 29.5 & 37.4 & 41.8 & 44.7 \\
\hline \multicolumn{6}{|l|}{ Of which } \\
\hline Locally incorporated banks & 53.7 & 51.1 & 60.0 & 65.1 & 67.5 \\
\hline \multicolumn{6}{|l|}{ Of which } \\
\hline Foreign banks & 2.0 & 5.7 & 6.3 & 6.4 & 10.2 \\
\hline Gross public sector exposure/total assets & 25.8 & 24.6 & 28.9 & 30.1 & 29.6 \\
\hline \multicolumn{6}{|l|}{ Of which } \\
\hline Locally incorporated banks & 38.5 & 30.0 & 37.8 & 43.2 & 44.3 \\
\hline \multicolumn{6}{|l|}{ Of which } \\
\hline Foreign banks & 9.2 & 16.3 & 15.0 & 10.3 & 8.9 \\
\hline \multicolumn{6}{|l|}{ Liquidity } \\
\hline Liquid assets to total assets & 41.7 & 43.4 & 37.3 & 36.9 & 35.2 \\
\hline \multicolumn{6}{|l|}{ Of which } \\
\hline Locally incorporated banks & 40.7 & 47.8 & 34.7 & 36.3 & 35.1 \\
\hline Liquid assets to short-term liabilities & 46.1 & 55.6 & 41.9 & 49.3 & 41.8 \\
\hline \multicolumn{6}{|l|}{ Of which } \\
\hline Locally incorporated banks & 48.2 & 81.4 & 44.4 & 60.9 & 46.7 \\
\hline \multicolumn{6}{|l|}{ Earnings and profitability } \\
\hline Return on average assets & 2.6 & 2.4 & 2.8 & 3.1 & 3.4 \\
\hline Return on average capital $1 /$ & 16.0 & 13.2 & 19.0 & 24.9 & 27.3 \\
\hline Interest margin to gross income & 47.9 & 48.8 & 40.2 & 39.5 & 36.6 \\
\hline Noninterest expenses to gross income & 29.1 & 33.4 & 26.7 & 26.5 & 24.1 \\
\hline \multicolumn{6}{|l|}{ Foreign exchange exposure } \\
\hline Net foreign exchange position/total capital $1 /$ & 1.1 & 1.3 & 1.1 & 1.0 & 1.1 \\
\hline
\end{tabular}

Source: Eastern Caribbean Central Bank.

1/ Capital ratios for locally incorporated commercial banks.

2/ Includes nonperforming loans, credits, and overdrafts (defined as being in arrears for 90 days or more). Nonperforming overdrafts are those on which no payments have been made for 90 days or credit is insufficient to cover interest charges. 
Table 20. St Kitts and Nevis: Balance of Payments

\begin{tabular}{|c|c|c|c|c|c|}
\hline & 2002 & 2003 & 2004 & 2005 & $\begin{array}{r}\text { Est. } \\
2006 \\
\end{array}$ \\
\hline \multicolumn{6}{|c|}{ (In millions of Eastern Caribbean dollars) } \\
\hline Current account & -370.5 & -341.1 & -217.5 & -267.7 & -388.1 \\
\hline Trade balance & -316.5 & -320.3 & -276.0 & -352.9 & -430.8 \\
\hline \multicolumn{5}{|l|}{ Of which } & 158.3 \\
\hline Sugar 1/ & 0.1 & 19.5 & 25.7 & 0.0 & 0.0 \\
\hline Imports f.o.b. & -479.5 & -474.2 & -434.2 & -500.1 & -589.2 \\
\hline \multicolumn{6}{|l|}{ Of which } \\
\hline Mineral fuel & -24.3 & -47.9 & -53.4 & -74.1 & -88.0 \\
\hline Services and transfers (net) & -54.0 & -20.8 & 58.5 & 85.3 & 42.7 \\
\hline Services net & 31.2 & 74.8 & 146.7 & 162.1 & 121.9 \\
\hline Services, receipts & 243.7 & 291.3 & 364.9 & 410.3 & 424.9 \\
\hline \multicolumn{6}{|l|}{ Of which } \\
\hline Tourism receipts & 154.3 & 203.5 & 277.1 & 309.2 & 314.1 \\
\hline Services, payments & -212.5 & -216.4 & -218.2 & -248.2 & -303.0 \\
\hline Factor income (net) & -129.6 & -145.8 & -137.4 & -132.4 & -133.4 \\
\hline \multicolumn{6}{|l|}{ Of which } \\
\hline Public sector interest & -45.3 & -55.8 & -57.3 & -60.7 & -59.8 \\
\hline Transfers (net) & 44.4 & 50.2 & 49.2 & 55.6 & 54.2 \\
\hline Official (net) & -1.1 & -1.2 & -3.6 & -2.7 & -3.9 \\
\hline Private (net) & 45.5 & 51.4 & 52.8 & 58.4 & 58.1 \\
\hline Capital and financial account & 357.5 & 302.6 & 238.2 & 149.6 & 384.5 \\
\hline Official & 143.1 & 147.6 & 61.9 & 39.1 & -0.2 \\
\hline Capital transfers (net) & 30.7 & 6.1 & 7.3 & 31.6 & 26.5 \\
\hline Long-term borrowing (net) & 112.3 & 141.5 & 54.6 & 7.5 & -26.7 \\
\hline Private capital & 214.4 & 155.0 & 176.3 & 110.5 & 384.7 \\
\hline Capital transfers (net) & 8.7 & 8.4 & 7.2 & 8.2 & 8.4 \\
\hline Foreign direct investment (net) & 215.4 & 204.2 & 124.6 & 230.8 & 539.1 \\
\hline Portfolio investment (net) & 18.2 & -1.1 & -62.8 & 9.0 & 9.0 \\
\hline Short-term capital (net) & -51.9 & -89.8 & 152.1 & -107.9 & -184.5 \\
\hline Other private (net) & 24.1 & 33.4 & -44.7 & -29.6 & 12.7 \\
\hline Errors and omissions & 42.5 & 36.6 & 17.1 & 99.6 & 49.8 \\
\hline Overall balance & 29.5 & -1.9 & 37.9 & -18.5 & 46.1 \\
\hline \multicolumn{6}{|c|}{ (In percent of GDP) } \\
\hline Current account & -39.1 & -34.9 & -20.2 & -22.6 & -29.0 \\
\hline Of which & & & & & \\
\hline Exports & 17.2 & 15.7 & 14.7 & 12.4 & 11.8 \\
\hline Imports & -50.6 & -48.5 & -40.3 & -42.3 & -44.1 \\
\hline Tourism receipts & 16.3 & 20.8 & 25.7 & 26.1 & 23.5 \\
\hline \multicolumn{6}{|c|}{ (Annual percentage change) } \\
\hline Merchandise exports (in U.S. dollars) & 9.7 & -5.6 & 2.8 & -7.0 & 7.6 \\
\hline Merchandise imports (in U.S. dollars) & 6.6 & -1.1 & -8.4 & 15.2 & 17.8 \\
\hline Terms of trade & 0.2 & 0.2 & -3.6 & -5.9 & -5.7 \\
\hline \multicolumn{6}{|l|}{ Memorandum items: } \\
\hline Outstanding external public debt $2 /$ & 75.9 & 88.5 & 85.5 & 77.0 & 66.9 \\
\hline \multicolumn{5}{|l|}{ Of which } & 25.1 \\
\hline Interest & 10.8 & 12.1 & 10.5 & 10.3 & 9.7 \\
\hline
\end{tabular}

Sources: Eastern Caribbean Central Bank; and Fund staff estimates.

1/ Sugar industry was closed in 2005.

2/ In percent of GDP, adjusted for valuation changes.

$3 /$ In percent of exports of goods and services. 
Table 21. St. Kitts and Nevis: Public Sector External Debt

\begin{tabular}{|c|c|c|c|c|c|}
\hline & 2002 & 2003 & 2004 & 2005 & $\begin{array}{r}\text { Est. } \\
2006 \\
\end{array}$ \\
\hline \multicolumn{6}{|c|}{ (In millions of Eastern Caribbean dollars) } \\
\hline \multicolumn{6}{|l|}{ Outstanding debt (end of period) $1 /$} \\
\hline Total public sector & 719.2 & 865.5 & 922.1 & 910.6 & 893.8 \\
\hline Central government & 494.8 & 643.6 & 663.2 & 596.1 & 600.6 \\
\hline Nonfinancial public enterprises & 172.3 & 163.9 & 194.4 & 238.4 & 212.4 \\
\hline Financial institutions & 52.1 & 58.0 & 64.5 & 76.1 & 80.8 \\
\hline \multicolumn{6}{|l|}{ Disbursements } \\
\hline Total public sector & 152.6 & 199.0 & 133.4 & 81.4 & 59.7 \\
\hline Central government & 134.1 & 187.1 & 62.9 & 7.5 & 45.2 \\
\hline Nonfinancial public enterprises & 15.0 & 4.4 & 62.4 & 63.1 & 0.0 \\
\hline Financial institutions & 3.5 & 7.5 & 8.1 & 10.8 & 14.5 \\
\hline \multicolumn{6}{|l|}{ Amortization (principal repayments) } \\
\hline Total public sector & 40.2 & 57.6 & 79.8 & 73.9 & 86.5 \\
\hline Central government & 26.3 & 42.1 & 45.3 & 55.6 & 49.8 \\
\hline Nonfinancial public enterprises & 11.3 & 12.9 & 31.9 & 15.0 & 26.1 \\
\hline Financial institutions & 2.6 & 2.6 & 2.6 & 3.3 & 10.5 \\
\hline \multicolumn{6}{|l|}{ Interest payments } \\
\hline Total public sector & 45.3 & 55.8 & 57.3 & 60.7 & 59.8 \\
\hline Central government & 31.6 & 43.5 & 46.1 & 47.4 & 41.7 \\
\hline Nonfinancial public enterprises & 11.7 & 10.3 & 9.3 & 11.0 & 12.1 \\
\hline Financial institutions & 2.0 & 2.0 & 2.0 & 2.4 & 6.1 \\
\hline \multicolumn{6}{|l|}{ Debt service } \\
\hline Total public sector & 85.6 & 113.4 & 137.2 & 134.6 & 146.3 \\
\hline Central government & 57.9 & 85.6 & 91.4 & 103.0 & 91.5 \\
\hline Nonfinancial public enterprises & 23.0 & 23.1 & 41.2 & 26.0 & 38.2 \\
\hline Financial institutions & 4.7 & 4.7 & 4.6 & 5.6 & 16.6 \\
\hline \multicolumn{6}{|c|}{ (In percent of GDP) } \\
\hline \multicolumn{6}{|l|}{ St. Kitts and Nevis public sector } \\
\hline Disbursements & 16.1 & 20.4 & 12.4 & 6.9 & 4.5 \\
\hline Amortization & 4.2 & 5.9 & 7.4 & 6.3 & 6.5 \\
\hline Interest payments & 4.8 & 5.7 & 5.3 & 5.1 & 4.5 \\
\hline Debt service & 9.0 & 11.6 & 12.7 & 11.4 & 10.9 \\
\hline Outstanding debt (end of period) & 75.9 & 88.5 & 85.5 & 77.0 & 66.9 \\
\hline Debt service (in percent of exports of goods and services) & 21.0 & 25.5 & 26.2 & 24.1 & 25.1 \\
\hline Effective interest rate (in percent per annum) & 6.9 & 7.0 & 6.4 & 6.6 & 6.6 \\
\hline \multicolumn{6}{|l|}{ Central government } \\
\hline Disbursements & 14.1 & 19.1 & 5.8 & 0.6 & 3.4 \\
\hline Amortization & 2.8 & 4.3 & 4.2 & 4.7 & 3.7 \\
\hline Interest payments & 3.3 & 4.5 & 4.3 & 4.0 & 3.1 \\
\hline Debt service & 6.1 & 8.8 & 8.5 & 8.7 & 6.8 \\
\hline Outstanding debt (end of period) & 52.2 & 65.8 & 61.5 & 50.4 & 44.9 \\
\hline Debt service (in percent of exports of goods and services) & 13.7 & 18.6 & 16.8 & 17.5 & 14.8 \\
\hline Effective interest rate (in percent per annum) & 7.2 & 7.6 & 7.1 & 7.5 & 7.0 \\
\hline
\end{tabular}

Sources: Ministry of Finance; Eastern Caribbean Central Bank; and Fund staff estimates.

1/ Adjusted for valuation adjustment. 